\title{
Optical Wireless Indoor Networks: Recent Implementation Efforts
}

\author{
Klaus-Dieter Langer and Jelena Vučić \\ Fraunhofer Institute for Telecommunications, Heinrich-Hertz-Institut, \\ Einsteinufer 37, 10587 Berlin, Germany; langer@fraunhofer.hhi.de
}

\begin{abstract}
The paper addresses concepts of recently implemented optical wireless transmission systems for broadband indoor applications based on infrared radiation as well as visible light. An overview of solutions and achieved performances is given.
\end{abstract}

\section{Introduction}

Even though behind the term 'wireless' conventionally radio technology is considered, both industry and scientific community agree that the next generation of wireless communication systems (4G) will be based on several complementary access technologies including optical wireless (OW) communications.

Wireless optical radiation offers attractive features, distinct from those of radio. First, there is huge, unregulated and available bandwidth, without electromagnetic interference (EMI) to existing radio systems. Moreover, OW signals do not penetrate through walls. Such signal confinement naturally makes each room a communication cell, eliminating any inter-cell interference. Thus, one can reuse the same operating frequencies in adjacent cells and hence, deploy uniform equipment in a cellular architecture. This enables a simple design of high capacity wireless local area networks since transmissions in adjacent rooms need not be coordinated. Due to the inherent high degree of privacy and security against eavesdropping, such systems are of interest e.g., for military use or financial messaging. Moreover, they are highly attractive for operating environments such as hospitals and aircrafts, where radio systems are illadvised. Thus, both radio and optical media might jointly provide a broad spectrum of capabilities that radio alone would find difficult to do.

Two basic link designs for indoor OW communication are the directed line-of-sight (LOS) and the non-directed link. The directed link (Fig. 1a) utilizes LOS for communication between a narrow-beam transmitter (Tx) and a narrow field-of-view (FOV) receiver (Rx). With available LOS and carefully aligned transceivers (TRx), high transmission speeds (beyond $100 \mathrm{Mbit} / \mathrm{s}$ ) can be achieved. In a non-directed link (Figs. 1b and c), the connection between a widebeam $\mathrm{Tx}$ and a wide FOV Rx relies on numerous signal reflections off the surfaces in the room, instead of, or in addition to the LOS. A link operating completely without LOS is referred to

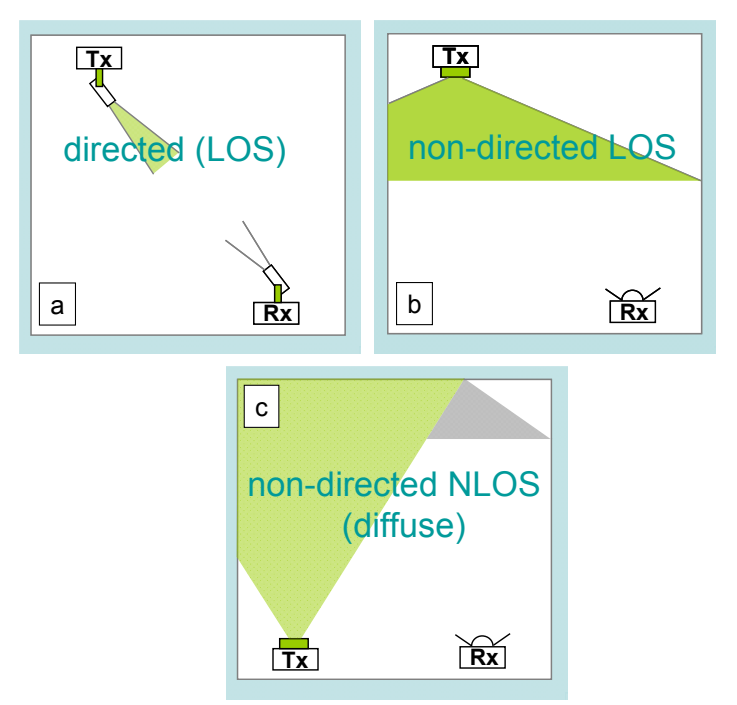

Fig. 1: Types of OW links: a) Directed line-of-sight (LOS), b) Non-directed LOS and c) non-directed non-LOS (diffuse)

as diffuse. The non-directed link is simpler than the directed one, more robust to shadowing and inherently supports user mobility. Nevertheless, due to multipath propagation effects, it can be severely speed-limited, and requires relatively large transmission power. A classification of systems with respect to link and receiver type was presented and discussed by Kahn et al. ${ }^{1}$

In practical applications, it is often desired to combine mobility of the diffuse and high-speed capability of the LOS systems. Recent implementation efforts towards $1 \mathrm{Gbit} / \mathrm{s}$ transmission with considerable coverage will be reviewed in the next section of this paper dealing with infrared (IR) systems.

The majority of OW systems is designed to operate in the near infrared at wavelengths either around $850 \mathrm{~nm}$ or in the range of $1550 \mathrm{~nm}$ (mainly due to existing optical sources and safety regulations). Since recently, however, communication via visible light (VLC) is gaining attention in R\&D, driven by progress on LED technology for solid-state lighting (400-780 nm). Novel high-power white LEDs possess clear 
advantages over conventional lighting sources (such as potentially high power efficiency and long lifetime expectancy) which make them a major candidate for the future lighting market. The potential synergy of illumination and data transmission functions by use of one optical source has stimulated numerous R\&D activities. ${ }^{2}$ Due to an ubiquitous lighting and signalling infrastructure, VLC can offer an additional service at comparably low extra cost. An overview of the technical constraints and challenges for VLC links can be found elsewhere. ${ }^{2}$

Undoubtedly, since data transmission is tightly related to the illumination function, VLC is attractive mostly in permanently illuminated environments - like large offices, industrial settings, medical areas, public transport, but even museums, supermarkets, etc. Our recent investigations have shown that the high optical power distributed via many LEDs (as typically used in LED-based lamps) in order to meet the requirements for illumination basically creates a LOS transmission channel. Such channel can support high data rates (>100 Mbit/s) with complete coverage of medium-sized rooms. ${ }^{3}$ Nevertheless, due to rather low modulation bandwidth of LEDs developed for illumination, most recent high-speed VLC prototypes reach transmission rates in the range of $100 \mathrm{Mbit} / \mathrm{s}$ for typical indoor distances. These are reviewed in the third section of this paper.

\section{Infrared systems}

Infrared optical wireless transmission has a long tradition. Typically, low-cost optical sources such as LEDs are used for low data rates and short-reach links. For high transmission rates, lasers are required, due to their high modulation bandwidth. On the other hand, lasers are point sources, and skin and eye safety regulations put stricter limitations on the emitted optical power. Usually, a diffuser is needed to render them safe.

In free-space optics, it is possible to achieve Gbit/s IR links operating over a few $\mathrm{km}^{\text {range }}{ }^{4}$ e.g., between buildings, but these rely on high transmit powers within a very narrow radiation angle. For indoor applications, apart from transmission rate, the focus is often also on link coverage. However, achieving a Gbit/s IR communication link with significant coverage is very challenging.

Traditionally, infrared efforts concentrated on short-range point-to-point links based on LOS. Commercially, such links are present for many years in low bit-rate, simplex remote control applications for domestic consumer electronics equipment, and are standardized by the Infrared Data Association (IrDA). Today, IrDA interfaces can be found also in a variety of portable devices, like mobile phones, laptop computers and personal digital assistants. In its most recent version (fast IrDA), the standard offers rates up to $16 \mathrm{Mbit} / \mathrm{s}$ (over approximately $1 \mathrm{~m}$ range), but interfaces for much higher data rates are in development (up to $1 \mathrm{Gbit} / \mathrm{s}$ with Giga-IR) ${ }^{5}$.

As mentioned in the introduction, LOS links are the most power- and bandwidth-efficient type, suitable for high-speed applications since the optical power is highly concentrated and there is no loss or pulse dispersion due to multipath propagation. However, precise transceiver alignment and susceptibility to shadowing limit their application to a specific environment. To enhance link coverage and support user mobility, different concepts have been proposed. Unfortunately, all these require rather complex multibeam-forming transmitters and angle diversity or self-orienting receivers. Subsequently, we present an (not necessarily complete) overview of recently implemented solutions and performances reported.

\section{Cellular communication scheme}

To secure robustness against connection failures in an indoor high-speed IR link, one can adopt a cellular communication scheme, where the coverage is increased by combining multiple narrow-beam links. Figure 2 shows a schematic of a typical cellular LOS system, implemented as part of the EU-FP7 project OMEGA ${ }^{6,7}$, which includes a base station and a number of user terminals. Both units have identical transceivers so that their FOVs have matching profiles.

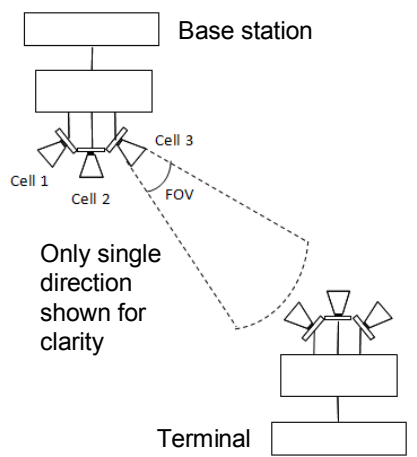

Fig. 2: LOS cellular IR communication system

At the transmitter side, input data is divided and used to modulate the laser sources in a seven-cell IR link. The receiver consists of three receiving modules that collect light from three directions (cells) simultaneously. Received signal strength indicators (RSSI) from all receivers (or cells) are then evaluated to determine which cell is currently available for communications. When the power level of the received signal at a given cell is greater than a pre-defined threshold (e.g., 

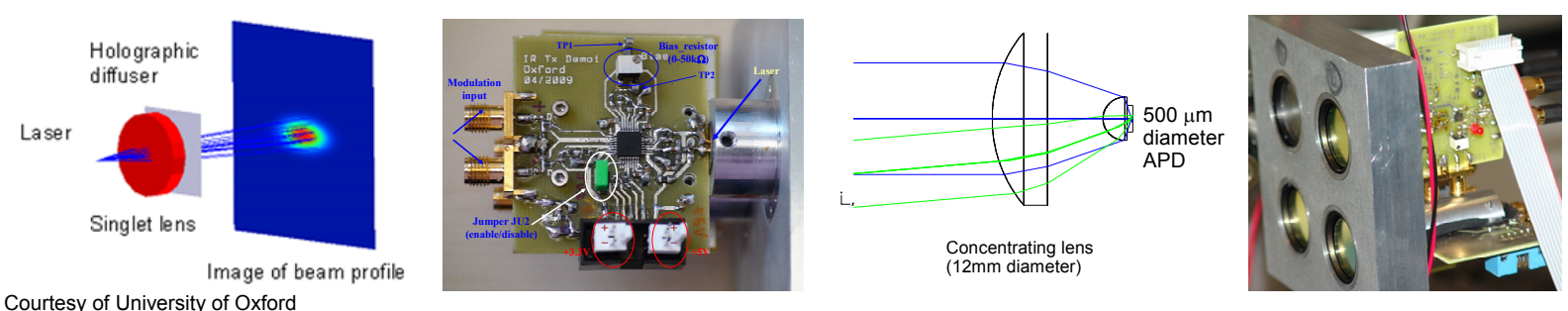

Fig. 3: Components of a $1.25 \mathrm{Gbit} / \mathrm{s}$ LOS cellular IR communications system (from left to right): Transmitter's optics design, laser driver, receiver's optics design and receiver's optoelectronics circuit

a bit error rate), the demultiplexer will route the received signal to the clock and data recovery unit. The data stream is then retimed and together with the recovered clock forwarded to the data layer. In the overlapped region of two cells the RSSI values of two receivers could be greater than the threshold level. Then, the receiver is randomly selected by means of a simple priority encoder. Although this approach does not select the receiver with the best signal power level, it offers a robust handover for initial demonstration purposes.

Figure 3 outlines the design and practical implementation of the Gbit/s optoelectronics subsystems for transmitter and receiver. The transmitter is designed such that it satisfies the laser class 1 standard. The system was calibrated and tested. ${ }^{8}$ It has been shown that a measured BER of $10^{-11}$ has been achieved for the $1.25 \mathrm{Gbit} / \mathrm{s}$ NRZ-OOK (on-off keying) link over $3 \mathrm{~m}$ distance in a coverage area of about $0.6 \mathrm{~m}^{2}$ with no forward error coding.

\section{Multispot diffuse (quasi-diffuse) configuration}

To improve the power-efficiency of diffuse links, the multispot diffuse configuration was suggested. ${ }^{9}$ These systems irradiate the walls and the ceiling with controlled radiation, and use a receiver that only accepts radiation from a limited range of angles. Figure 4 shows the geometry of such systems, where a multiple-beam source is used in combination with an array of narrow FOV receivers that each 'see' one spot. The spot is not dispersive as all the path lengths from transmitter to receiver are close to the same distance. The same work ${ }^{9}$ introduced the concept of the imaging receiver to achieve this function.

Recently, a measurement setup was implemented, to characterize the channel of the quasi-diffuse link using near-IR wavelengths. Figure 5 shows a photograph of the experimental setup. The laser transmitter has a collimating lens attached to it, which allows focusing a spot on the ceiling, where the IR light gets diffused. The reflected light is captured with a focusing lens at the receiver. The obtained frequency re-

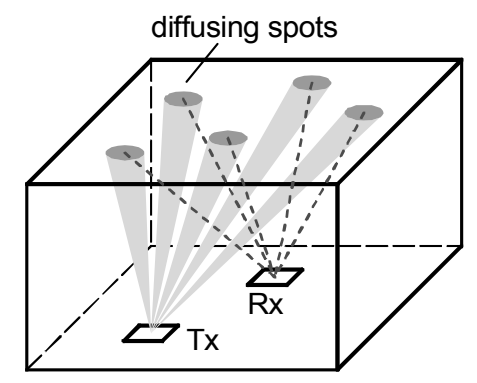

Fig. 4: Multispot diffusing configuration using a multi-beam transmitter

sponse plots revealed an approximately flat OW channel characteristic up to $1 \mathrm{GHz}$. The implication of this finding is that the channel contains very little multipath distortion, and would allow transmission of data with little Inter-Symbol Interference (ISI), if the signaling rate is 2 Gigasymbols per second ${ }^{10}$, assuming a sufficient power budget.

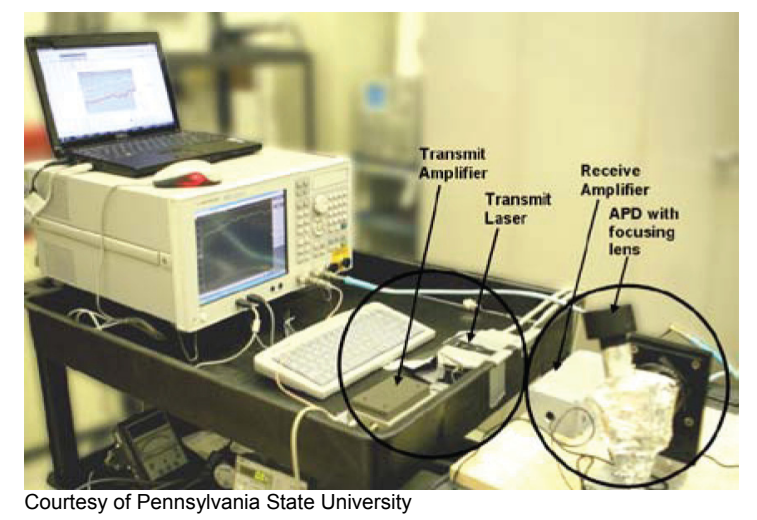

Fig. 5: Setup for indoor OW channel characterization

\section{Visible light systems}

Key component in VLC systems is an LED, radiating in the visible part of the electromagnetic spectrum. Most promising candidate for future general lighting is the phosphorescent white LED, which consists of a blue LED chip covered with a layer of yellow phosphor. Therefore, most of the VLC investigations are based on this device. Phosphorescent white LEDs are low-cost 
and have simpler driving, but they also have a rather poor modulation bandwidth (lower $\mathrm{MHz}$ range). The slow response results mainly from the phosphor component. One of the most successful approaches to significantly enhance the modulation bandwidth is to suppress the $\mathrm{Ph}$ affected portion of the optical spectrum at the receiver, by placing a suitable optical filter ("blue" filter) in front of the detector.

The "blue-filtering" approach was first suggested by Grubor et al. ${ }^{11}$, where $100 \mathrm{Mbit} / \mathrm{s}$ were achieved in a lab proof-of-concept, using discrete multitone (DMT, i.e. orthogonal frequency division multiplexing, OFDM, as it is known from mobile radio systems). DMT is a highly spectrally efficient modulation and multiplexing technique, which can be of great advantage when high transmission rates are targeted in bandwidth limited systems (such as VLC). A very valuable DMT property is that one can perform bit- or power-loading, and in that way efficiently exploit the channel capacity, i.e. enhance system flexibility and performance robustness. As any multiple-subcarrier-based modulation technique, DMT in an OW system requires a dc bias that carries no information. However, in VLC, dc is anyhow required for illumination and therefore, it is not causing any severe loss of powerefficiency.

The highest transmission speeds reported so far in literature using phosphorescent white LEDs and blue filtering are $200+\mathrm{Mbit} / \mathrm{s}(\mathrm{OOK})^{12}$ and $230 \mathrm{Mbit} / \mathrm{s}(\mathrm{DMT})^{13}$.

\section{Low-speed VLC prototypes}

Little et al. ${ }^{14}$ at Boston University demonstrated a low-rate $(\sim 50 \mathrm{kbit} / \mathrm{s})$ short-range $(\sim 3 \mathrm{~m})$ duplex point-to-point white-LED system developed with readily-available electronics and LEDs. OOK was applied to modulate the LED array. The aim of this prototype was to demonstrate viability, simplicity, and low cost of VLC solutions rather than their upper bound in terms of achievable data rates. Later on, the same team created a prototype that delivers in excess of $1 \mathrm{Mbit} / \mathrm{s}$

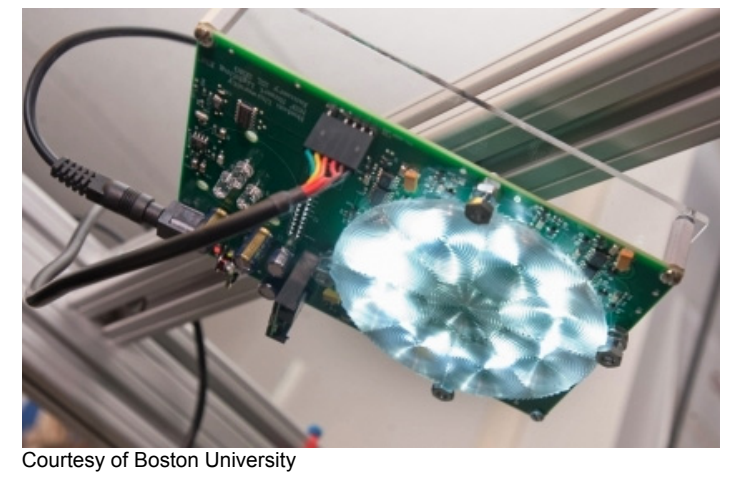

Fig. 6: Smart lighting prototype for R\&D applications while providing both illumination and communication at several meters and has been demonstrated as an array of seven luminaries in the form of overhead spot lighting. The latest prototype of a VLC transceiver with an array of nine high-brightness white LEDs and an array of three photodiodes is shown in Fig 6.

Another proof-of-principle implementation effort was done by a group at Jacobs University Bremen, Germany. Here, an indoor broadcasting system was realized by modulation of white LEDs with OFDM signals. ${ }^{15}$

The Tx hardware prototype consisted of a digital signal processing (DSP) development board to generate the OFDM analogue signal and the analogue front-end which includes an array of nine LEDs and the driver electronics. The Rx consisted of a PD and a preamplifier, whereas OFDM demodulation was implemented on another DSP board.

Due to the choice of the DSP board, the OFDM signal bandwidth was limited to a somewhat low value of $45 \mathrm{kHz}$. Here again, the target of the work was not to showcase high data rates, but rather to conduct studies via a simple proof-of-concept hardware demonstrator. Differently to the approaches with blue-filtering, the whole white-light radiation range was demodulated.

The conducted studies have shown that at $\mathrm{Tx}-\mathrm{Rx}$ distances of $1 \mathrm{~m}$ (typical for a reading lamp), with faster data converters and analogue front-ends supporting $20 \mathrm{MHz}$ (as e.g. with the blue-filtering approach), throughputs of about $80 \mathrm{Mbit} / \mathrm{s}$ should be feasible at illuminance levels in the recommended range. Indeed, this was confirmed by the hardware prototype developed within the OMEGA project, as is reported below.

In order to achieve prototypes operating in the range of $100 \mathrm{Mbit} / \mathrm{s}$, different approaches were followed to enhance the modulation bandwidth of white LEDs (as e.g., blue filtering) in combination with simple OOK, but also with aforementioned spectrally efficient modulation and multiplexing techniques (DMT, i.e. OFDM).

\section{Analogue pre-equalisation plus blue filtering}

At University of Oxford, the use of analogue equalization in white-LED-based VLC links has been investigated. As arrays of LEDs are envisaged for lighting, each device can be individually equalized in a way that the entire array provides much higher bandwidth than its single devices (see Fig. 7). This approach was first presented by Le Minh et al. $^{16}$, where a bandwidth of $25 \mathrm{MHz}$ was achieved. The configuration consisted of an array of 16 LEDs, where each device had a modulation bandwidth of $3 \mathrm{MHz}$. A 


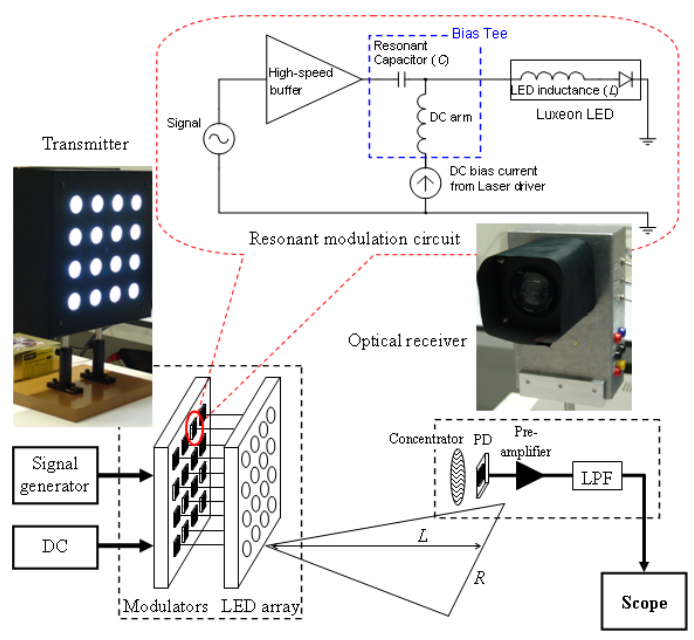

Courtesy of University of Oxford

Fig. 7: Visible light wireless transmission link using resonant driving of a $4 \times 4$ LED array

transmission rate of $40 \mathrm{Mbit} / \mathrm{s}$ over a distance of $2 \mathrm{~m}$ was demonstrated using NRZ-OOK. By combining the analogue equalization of a single link with blue filtering, $100 \mathrm{Mbit} / \mathrm{s}$ was achieved over a short distance of $\sim 10 \mathrm{~cm} .{ }^{17}$

High-speed video streaming via white LED

Within the EU Project OMEGA ${ }^{6}$, partners have shown for the first time an implementation of a real-time DMT-based visible-light link operating at $100 \mathrm{Mbit} / \mathrm{s}$ using a single low-cost off-theshelf white LED module. Modulation bandwidth of the LED was enhanced by blue-filtering ${ }^{3}$. The LED driver limited the system bandwidth to $\sim 10 \mathrm{MHz}^{18}$, so spectrally efficient DMT modulation was implemented.

The analogue front-ends were based on simple low-cost commercially available components. Digital signal processing was implemented in FPGA. The DMT signal consisted of 31 electrical subcarriers (excluding unmodulated dc) within a baseband bandwidth of $30.6 \mathrm{MHz}$, all modulated with 16-QAM. Due to the modulation significantly beyond the analogue system bandwidth, power pre-equalization (in digital domain, in front of the IFFT block) was introduced in order to enforce similar transmission quality (SNR) on all modulated subcarriers. Implementation of a standard Reed-Solomon forward error correction (FEC) mechanism rendered error-free transmission for illuminance levels in the range recommended for working environment ${ }^{19}$.

Demonstration on application level was performed by showing an error-free transmission of a video stream over the VLC link. MAC layer functionalities were specifically adapted for VLC broadcast links and also implemented in FPGA. ${ }^{20}$ Video transmission was demonstrated

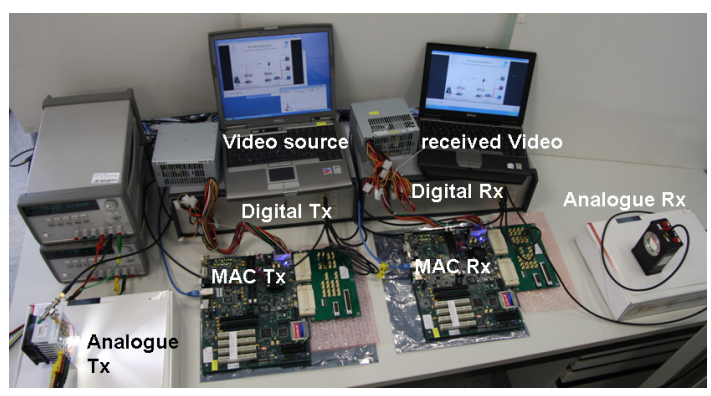

Fig. 8: Photograph of $100 \mathrm{Mbit} / \mathrm{s}$ VLC link demonstration by video streaming

over a distance of $1.4 \mathrm{~m} \mathrm{(} 500 \mathrm{~lx}$, enough to read and write $\left.{ }^{19}\right)$. A photograph of the complete VLC prototype is presented in Fig. 8. On the Tx side (left), a video stream hosted on a lap-top was fed into the system, and at the Rx side (right), the received video was displayed on another lap-top. All the main system components are pointed out in the figure. More details about this demonstrator can be found in Vučić et al. ${ }^{21}$

\section{High-speed full-duplex LED-based system}

The group from Nakagawa Laboratiories, Japan implemented a $100 \mathrm{Mbit} / \mathrm{s}$ full-duplex multiple access system, where downlink and uplink transmission are separated by using two different wavelength windows: white light for downlink and infrared $(850 \mathrm{~nm})$ for uplink. Support for multi-user access was realized by an optical CSMA/CD (carrier sense multiple access with collision detection) method, in order to ensure compatibility with wireline LAN communications such as Ethernet networks or PLC. Shared access was provided to a group of user terminals in the way that data are only sent when no carrier collision occurred and the physical medium was idle.

The system consists of one non-directed access point and several directed optical end-terminals as illustrated by Fig. 9. ${ }^{22}$ The access

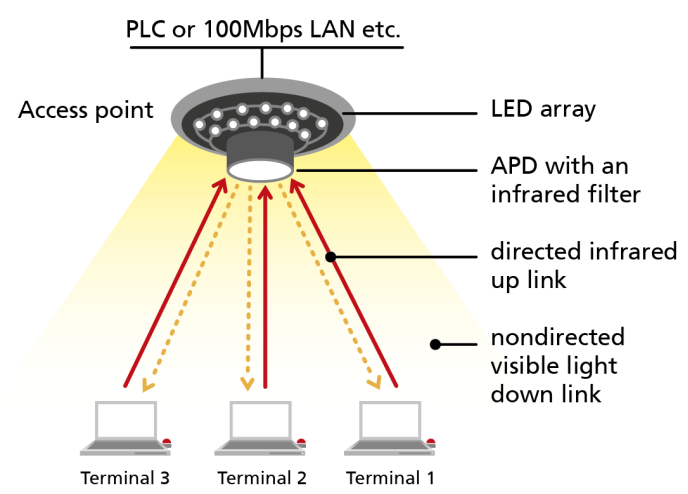

Fig. 9: Schematic diagram of a full-duplex multiaccess system based on (white and infrared) LED modulation 
point has a white LED array transmitter of toric shape and an IR APD receiver (including a visible-light cut filter in its entrance window). It provides a service area for communications and illumination of about $9 \mathrm{~m}^{2}$ at $1.7 \mathrm{~m}$ distance below the ceiling (desk-top height). Network layer and CSMA/CD functionalities were implemented in FPGA.

The bidirectional user terminal consists of an IR-LED Tx and an IR-suppressing APD Rx. The Tx is a pair of LEDs, with separate lenses for focussing the dispersed LED radiation to the access point. Networking functions of the user terminal were again implemented in FPGA.

\section{Conclusions}

Increased demands on wireless communication on one hand and the fact that the limited radio frequency spectrum gets more and more crowded on the other, resulted in high interest in optical wireless transmission technology for indoor applications.

Recent lab demonstrations, as well as prototype implementations of several independent groups, demonstrated both the feasibility of high-capacity full-duplex infrared hot-spots up to $1 \mathrm{Gbit} / \mathrm{s}$ and practically entirely covered rooms when using VLC broadcast at data rates up to $100 \mathrm{Mbit} / \mathrm{s}$. Significant work has been done on both the PHY as well as the MAC layers. Related standardization activities are in progress by bodies such as IrDA, IEEE and Visible Light Communications Consortium (VLCC) ${ }^{23}$.

Feasibility of IR communications at multiGbit/s speeds remains challenging due to the low link power budget. A lot of technological advances are necessary to achieve low-cost user friendly (i.e. spatial coverage) systems which at the same time offer high transmission speeds (permanently or at least dynamically adapted to the present channel conditions).

Performance of the current high-speed VLC prototypes is limited mostly by the bandwidth and imperfect linearity of the LED module and its driver, so high bit rates are aspired by spectrally-efficient modulation as well as equalization (in analogue and/or digital domain). Results of our most recent lab experiments indicate a possibility for significant further performance enhancement in the course of ongoing work. ${ }^{24}$

\section{Acknowledgement}

We would like to thank D. O'Brien, M. Kavehrad, S. Haruyama, T.D.C. Little, H. Haas and H. Le Minh for valuable inputs and remarks. We are grateful to our colleagues from $\mathrm{HHI}$ and partners from the OMEGA project. Parts of the author's work presented in this paper got funding from the EU-FP7 projects OMEGA and BONE.

\section{References}

1 J.M. Kahn et al., IEEE Communications Magazine 12 (1998).

2 D. O'Brien et al., Visible light communications, Kraemer and Katz (Eds.), Short-range wireless communications, J. Wiley (2009).

3 J. Grubor et al., IEEE JLT 26, 3883 (2008).

4 G. Nykolak et al., El. Letters 35, 578 (1999).

5 Infrared Data Association, www.irda.org/ associations/2494/files/Giga-IR General.pdf (2009).

6 EU-FP7 project OMEGA www.ict-omega.eu/ (2010).

7 H. Le Minh et al., Proc. ICTON '10, Th.A3.1 (2010).

8 H. Le Minh et al., Proc. CSNDSP '10, OWC15 (2010).

9 G. Yun, M. Kavehrad, Proc. IEEE Sel. Topics in Wireless Commun., 262 (1992).

10 M. Kavehrad, J. Fadlullah, Proc. SPIE $\mathbf{7 6 2 0}$ (2010).

11 J. Grubor et al., Proc. ECOC '07, PD3.6 (2007).

12 J. Vučić et al., Proc. OFC '10, OThH3 (2010).

13 J. Vučić et al., IEEE PTL 21, 1511 (2009).

14 T.D.C. Little et al., Proc. WiMob '08 (2008).

$15 \mathrm{H}$. Elgala et al., Trans. Consumer Elect. 55, 1127 (2009).

$16 \mathrm{H}$. Le Minh et al., IEEE PTL 20, 1243 (2008).

$17 \mathrm{H}$. Le Minh et al., IEEE PTL 21, 1063 (2009).

18 R. Baumgartner et al., IEEE P802.15.7 TGVLC (2010).

19 European standard EN 12464-1: Lighting of indoor work places (2003).

20 ICT-OMEGA Deliverable D4.3, Opt. Wireless MAC-LLC Spec., www.ict-omega.eu/ (2009).

$21 \mathrm{~J}$. Vučić et al., to appear in Proc. ECOC '10 (2010).

22 X. Lin et al., Proc. IMETI '09 3, 275 (2009).

23 Visible Light Communications Consortium, ww.vlcc.net/ (2010).

24 ICT-OMEGA news march 2010, www.ictomega.eul (2010). 